\title{
ON THE EXCITATION OF OSCILLATIONS OF THE SUN (NUMERICAL MODELS)*
}

\author{
A. G. KOSOVICHEV and A. B. SEVERNY \\ Crimean Astrophysical Observatory, p/o Nauchny, 334413, Crimea, U.S.S.R.
}

\begin{abstract}
Numerical solutions of the general time-dependent gas-dynamical equations in linear adiabatic approximation are given for initial conditions imitating: (a) a central perturbation, (b) a boundary perturbation (in the convective envelope), and (c) a 'shrinking' of the Sun as a whole. For a variety of models of the Sun it is found that at the surface the radial component $v_{r}$ of velocity is much greater than the tangential component $v_{t}$, and that the period $T$ of stationary oscillations does not exceed $131^{\mathrm{m}}$. The appearance at the surface of a $g$ mode with period $160^{\mathrm{m}}$ is found to be improbable.

With the initial conditions adopted, a propagating wave is produced which is reflected successively from the centre to the periphery and back, producing 5-min oscillations at the surface of the Sun. Expansion of this wave into separate modes leads to a power spectrum qualitatively similar to that observed.
\end{abstract}

\section{Introduction}

$160^{\mathrm{m}}$ oscillations can be obtained as an eigenmode of a standard model of the Sun only among higher order $g$ modes $(n=9-12$ for $l=2$; e.g., Christensen-Dalsgaard and Gough, 1976; Iben and Mahaffy, 1976; Vorontzov and Zharkov, 1978; and others). This is difficult to reconcile with the fact that usually lower modes are first and most easily excited. Therefore an explanation in terms of $g$ modes seems to be artificial. Lower-order modes $\left(g_{2}-g_{4}\right)$ might explain the $160^{\mathrm{m}}$ periodicity if the Sun has suffered permanent or transient mixing (e.g. model $H$ of Christensen-Dalsgaard and Gough, 1980; Schatzman et al., 1981, model with turbulent diffusion; and Christensen-Dalsgaard et al., 1974, transiently mixed models). It is noteworthy that these mixed models also offer a possible explanation of the low output of neutrinos from the Sun.

Since, in our opinion, the problem of solar oscillations should be coupled with the problem of their excitation, we have taken a different approach from previous authors, and have considered numerically which oscillations can result from different types of excitation. The latter we represent by different initial perturbations.

\section{Statement of the Problem}

The general equations of motion, in linear adiabatic approximation, are:

$$
\frac{\partial \mathbf{v}}{\partial t}=-\frac{1}{\rho_{0}} \nabla p^{\prime}+\frac{\rho^{\prime}}{\rho_{0}^{2}} \nabla p_{0}+\nabla \phi^{\prime},
$$

\footnotetext{
* Proceedings of the 66th IAU Colloquium: Problems in Solar and Stellar Oscillations, held at the Crimean
} Astrophysical Observatory, U.S.S.R., 1-5 September, 1981. 


$$
\begin{aligned}
& \frac{\partial \rho^{\prime}}{\partial t}+\operatorname{div}\left(\rho_{0} \mathbf{v}\right)=0, \\
& \frac{\partial p^{\prime}}{\partial t}-\gamma \frac{p_{0}}{\rho_{0}} \frac{\partial \rho^{\prime}}{\partial t}+\left(\frac{\mathrm{d} p_{0}}{\mathrm{~d} r}-\gamma \frac{p_{0}}{\rho_{0}} \frac{\mathrm{d} \rho_{0}}{\mathrm{~d} r}\right) v_{r}=0, \\
& \nabla^{2} \phi^{\prime}=-4 \pi G \rho^{\prime} .
\end{aligned}
$$

These have been solved numerically for polytropes of indices $n=0,1.5$, and 3, and for several more realistic models of the Sun: the standard model of Dziembowski and Pamyatnykh (1978), model $H$ of Christensen-Dalsgaard and Gough (1980), and the model of Schatzman et al. (1981) with turbulent diffusion. The boundary conditions are as usual: the regularity condition at the centre $r=0$ (when relevant) and the absence of external forces and the continuity of gravitational potential and its first derivative at the photosphere $r=R_{\odot}$.

Time-dependent computations from an initial state of rest that is perturbed slightly from the equilibrium configuration were performed. Three kinds of initial perturbation were considered:

(a) one localized near the centre (imitating a fluctuation in energy generation):

$$
\begin{aligned}
& \frac{\rho^{\prime}}{\rho_{0}}=\varepsilon\left(\frac{r}{a}\right)^{\prime} \exp \left[-0.5\left(\frac{r-r_{0}}{a}\right)^{2}\right] P_{l}(\cos \theta), \quad \frac{p^{\prime}}{p_{0}}=\gamma \frac{\rho^{\prime}}{\rho_{0}} ; \\
& \varepsilon=10^{-10}, \quad a=0.1 R_{\odot}, \quad r_{0}=0 ;
\end{aligned}
$$

(b) one localized near the surface (imitating a perturbation in the convection zone), which is of the same form as (2) but with

$$
\varepsilon=10^{-9}, \quad a=0.05 R_{\odot}, \quad r_{0}=0.85 R_{\odot} ;
$$

(c) a perturbation distributed throughout the body of the Sun (imitating an overall 'shrinking'):

$$
\begin{aligned}
& r^{\prime}=r-\xi(r), \quad \xi(r)=\xi_{0}\left(r / R_{\odot}\right)^{2} P_{2}(\cos \theta), \\
& \frac{\rho^{\prime}}{\rho_{0}}=-\frac{1}{\rho_{0}} \xi(r) \nabla \rho_{0},
\end{aligned}
$$

with

$$
\xi_{0}=1 \mathrm{~m}
$$

The excitation of oscillations by a pulsating core of radius $0.1 R_{\odot}$ with periods $7^{\mathrm{m}}-160^{\mathrm{m}}$ has also been considered. In this case the perturbation is expressed as a boundary condition at $r=0.1 R_{\odot}$ (see Kosovichev and Severny, 1981). 


\section{Results}

Results of the calculations for quadrupole oscillations of the $n=3$ polytrope for cases (a), (b), (c) are presented on Figures 1 to 3, respectively. Plotted is the response to the initial conditions near the centre, at $r=0.1 R_{\odot}$, and near the surface, at $r=0.995 R_{\odot}$.

In case (a) the central perturbation produces a wave with amplitude $v_{r}=$ $=1.4 \times 10^{-5} \mathrm{~m} \mathrm{~s}^{-1}$ propagating outwards to the surface, at which the amplitude has increased to $0.24 \mathrm{~m} \mathrm{~s}^{-1}$. Then the wave is reflected back to the centre. The successive reflections from the centre and the surface lead to a stationary regime of oscillations with a period $T$ of successive appearances of the perturbation at the surface given by

$$
T=2 \int_{0}^{R_{0}} \frac{\mathrm{d} r}{c_{s}}=131^{\mathrm{m}} .
$$

An important result is that near the surface $v_{r} \gg v_{r}$ : radial motion, apparently associated with $p$ modes, predominates. In the central regions, however, $v_{r} \sim v_{t}$, which suggests the presence of $g$ modes.

In case (b) two waves appear, one propagating to the centre, and the other to the surface. In this case the resulting pattern of oscillations is qualitatively similar to the first.

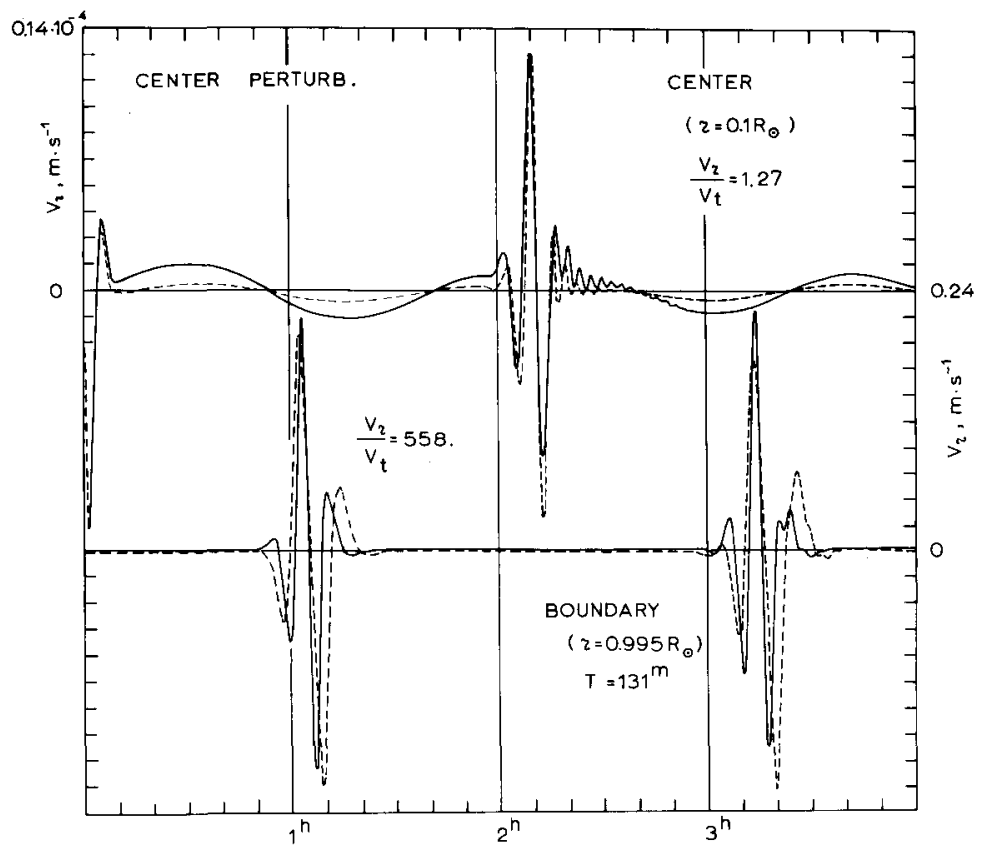

Fig. 1. Evolution of the radial velocity $v_{r}$ (solid line) and the tangential velocity $v_{t}$ (dashed line) near the centre of the Sun $\left(r=0.1 R_{\odot}\right)$ and near the surface $\left(r=0.995 R_{\odot}\right)$, after the initial perturbation (a) with $l=2$. 


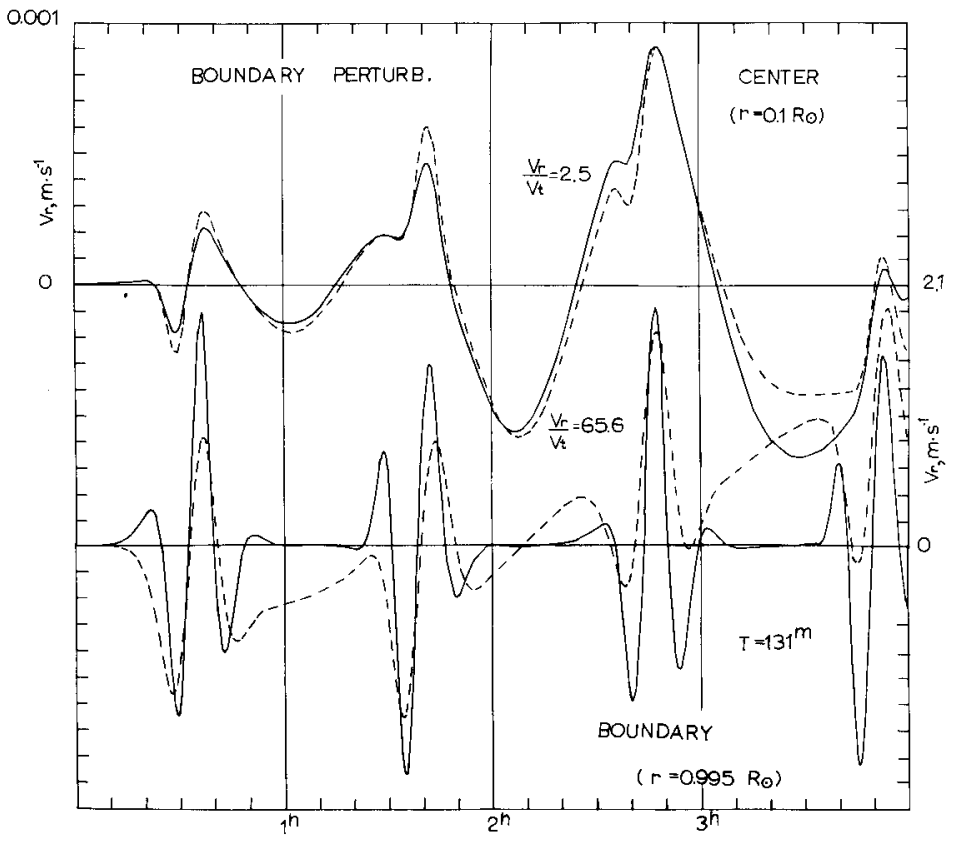

Fig. 2. Similar to Figure 1, but for initial perturbation (b).

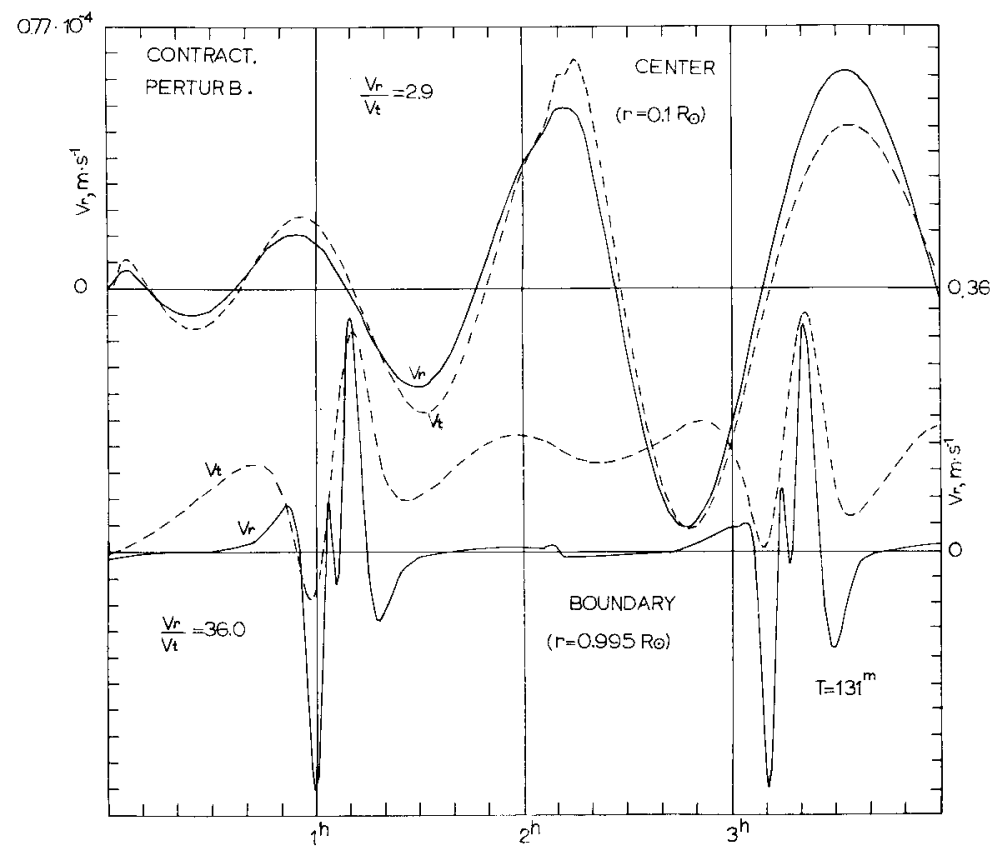

Fig. 3. Similar to Figure 1, but for initial perturbation (c). 
The surface amplitude of $v_{r}$ is $2.1 \mathrm{~m} \mathrm{~s}^{-1}$, and $v_{r} \gg v_{t}\left(v_{t}=3.2 \times 10^{-2} \mathrm{~m} \mathrm{~s}^{-1}\right)$. Again $T=131^{\mathrm{m}}$. A similar behaviour is found to result from perturbations of type (c) too.

We have found that this behaviour is exhibited also by all the solar models we have considered (excluding the unstable $n=0$ polytrope, for which we obtained a period of $187^{\mathrm{m}} .2$, which is equal to that of the Kelvin mode of an incompressible fluid sphere (Thomson, 1863), and a characteristic growth time of $31^{\mathrm{m}} .7$ for the $g_{1}$ mode, agreeing with that obtained by Pekeris (1938) for the compressible sphere). Oscillation periods vary from $114^{\mathrm{m}}$ for the Schatzman and Maeder model and $117^{\mathrm{m}}$ for model $H$ of Christensen-Dalsgaard and Gough (1980), up to $131^{\mathrm{m}}$ for the $n=3$ polytrope.

By considering the response to perturbations of types (a), (b), and (c) we do not find a dominant component of the resultant motion with a period of $160^{\mathrm{m}}$. Moreover, it is always the case that $v_{r} \gg v_{t}$ at the surface (except in the case of the polytrope with $n=0$ ). In the terminology of eigenmodes, we can say that at the surface of the Sun $p$ modes are dominant for the perturbations we have considered. We infer that one should observe mainly radial oscillations of the solar surface. That is indeed just what is observed (our attempts to identify transverse modes have not yet succeeded).

\section{Discussion}

The foregoing analysis raises the question of whether we are likely to detect low-frequency $g$ modes at the surface in general? In all the calculations considered above, low-frequency $g$ modes are confined to the central regions of the Sun, and at the surface the motion is dominated by $p$ waves and their wakes (see Kosovichev and Severny, 1981). Whether $g$ modes can appear at the surface is a question that should be considered.

To answer this question we calculated $l=2 \mathrm{~g}$ modes for the $n=3$ polytrope, paying particular attention to $g_{4}$, whose period $T=148^{\mathrm{m}} \cdot 6$ is the nearest to $160^{\mathrm{m}}$. (We found that our time-dependent programme reproduces all the $g$ modes perfectly, in complete agreement with earlier calculations by Vandakurov (1967) and Robe (1968).) Then we considered the following problem: assuming that this $g_{4}$ mode is present initially with $v_{r}=1 \mathrm{~m} \mathrm{~s}^{-1}$ at $r=R_{\odot}$, can it dominate at the surface after the introduction of some additional perturbation? The answer, as our calculations show, is pessimistic: namely, the $g_{4}$ mode contributes substantially to $v_{r}$ only if a central perturbation of type (a),

$$
\frac{\rho^{\prime}}{\rho_{0}}=\varepsilon\left(\frac{\rho^{\prime}}{\rho_{0}}\right)_{g_{4}}\left(\frac{r}{a}\right)^{2} \exp \left(-0.5 \frac{r^{2}}{a^{2}}\right) P_{2}(\cos \theta),
$$

has a relative amplitude $\varepsilon$ smaller than $0.1 \%$. For the perturbation near the boundary of type (b), $\varepsilon$ must be less than $1 \%$.

Figure 4 illustrates the result for case (a). The pattern of the radial component of velocity consists of bursts of oscillations with period $131^{\mathrm{m}}$, with large peak amplitude $v_{r, p}$, superposed on the slower $148^{\mathrm{m}} \cdot 6$ oscillation associated with the original $g_{4}$ mode. The latter has amplitude $v_{r, g}$ comparable with $v_{t, g}$, which is only about one tenth of $v_{r, p}$.

Our computations do not explain how the $160^{\mathrm{m}}$ period can appear, because all the 


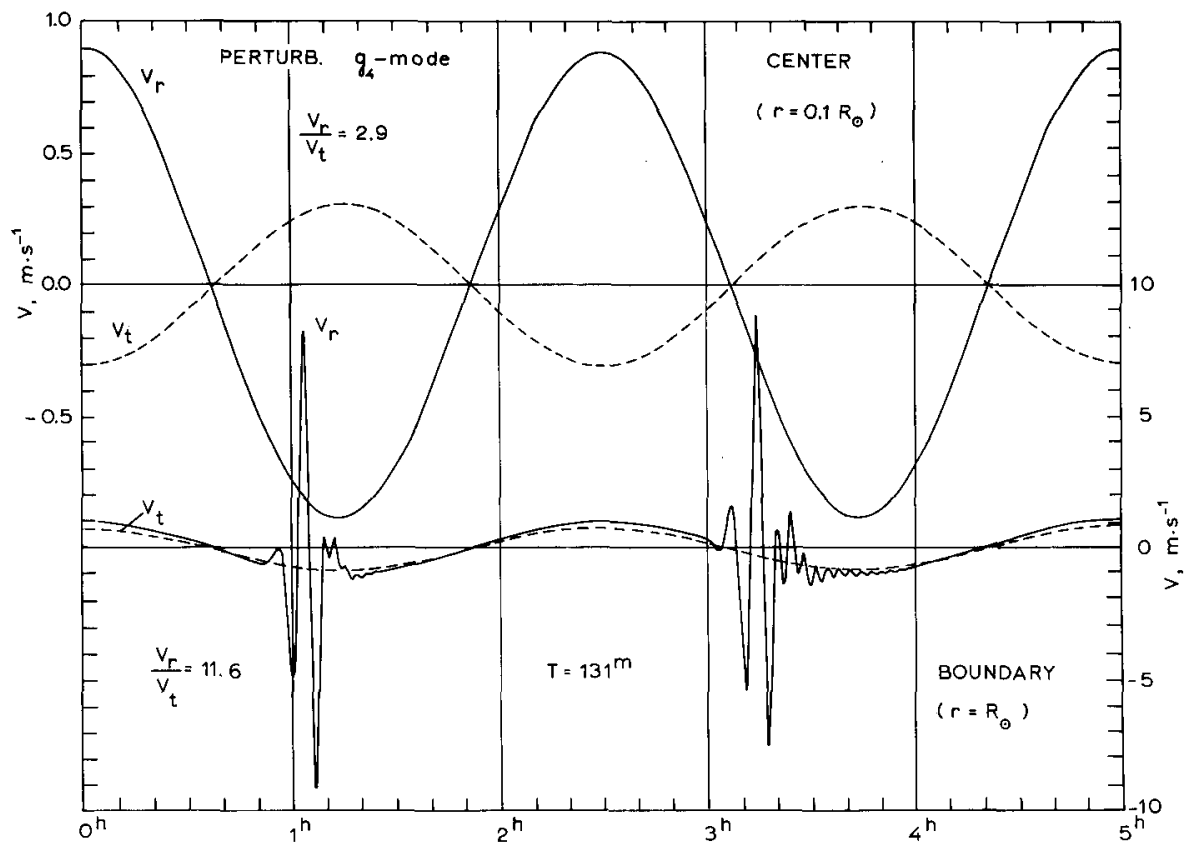

Fig. 4. Similar to Figure 1, but with $g_{4}(l=2)$ present initially together with perturbation $(8)$ with $\varepsilon=0.1 \%$.

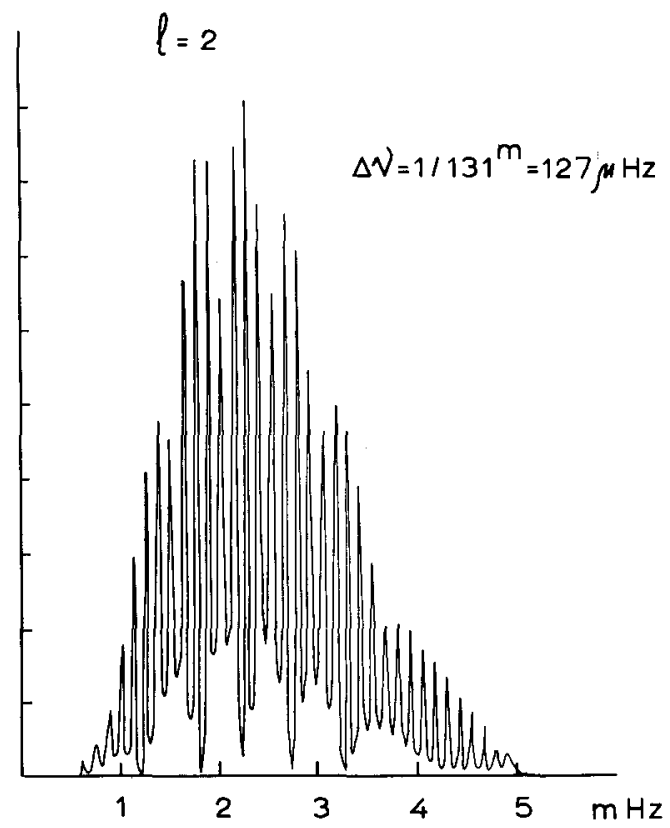

Fig. 5. Power spectrum near $3 \mathrm{mHz}$ of quadrupole oscillations of the surface of the $n=3$ polytrope following an initial perturbation of type (a). 
perturbations we considered lead to the appearence of oscillations like $p$ modes with periods $\lesssim 131^{\mathrm{m}}$. The $160^{\mathrm{m}}$ oscillations that are evident at the surface of the Sun indicate that perturbations inside the Sun of the type we have considered are not very strong. Otherwise $p$ modes would predominate, and we would see only the higher-frequency oscillations.

The power spectrum of solar oscillations at the surface for each $l$ and $n=3$ has, besides the peak corresponding to $T=131^{\mathrm{m}}$, a set of discrete equidistant peaks in the region around $3 \mathrm{mHz}$, with a spacing $\Delta v=1 / 131^{\mathrm{m}}=127 \mu \mathrm{Hz}$ (see Figure 5). This power spectrum is qualitatively very similar to those found from whole-disk measurements (see Claverie et al., 1979; Grec et al., 1980).

\section{Acknowledgements}

We are gratefull to Dr D. O. Gough for reading and considerable improvement of the manuscript.

\section{References}

Christensen-Dalsgaard, J. and Gough, D. O.: 1976, Nature 259, 89.

Christensen-Dalsgaard, J. and Gough, D. O.: 1980, Nature 288, 544.

Christensen-Dalsgaard, J., Dilke, F. W. W., and Gough, D. O.: 1974, Monthly Notices Roy. Astron. Soc. 169, 429.

Claverie, A., Isaak, G. R., Mcleod, C. P., van der Raay, H. B., and Roca Cortes, T.: 1979, Nature $282,591$.

Dziembowski, W. and Pamyatnykh, A. A.: 1978, in J. Rösch (ed.), Plein feux sur la physique solaire, CNRS, Paris, p. 135.

Grec, G., Fossat, E., and Pomerantz, M.: 1980, Nature 288, 541.

Kosovichev, A. G, and Severny, A. B.: 1981, Astron. Zh. Pisma 7, 34.

Iben, I. and Mahaffy, J.: 1976, Astrophys. J. 209, L39.

Pekeris, C. L.: 1938, Astrophys. J. 88, 189.

Robe, H.: 1968, Ann. Astrophys. 31, 475.

Schatzman, E., Maeder, A., Angrand, F., and Glowinski, R.: 1981, Astron. Astrophys, 96, 1.

Thomson, W.: 1863, Phil. Trans. Roy. Soc. London 153, 583.

Vandakurov, Yu. V.: 1967, Astron. Zh. 44, 786.

Vorontsov, S. V. and Zharkov, V. N.: 1968, Astron. Zh. 55, 84. 